University of Nebraska - Lincoln

DigitalCommons@University of Nebraska - Lincoln

Timothy J. Gay Publications

Research Papers in Physics and Astronomy

October 1996

Current Status Of The Saddle-Point Model

Timothy J. Gay

University of Nebraska - Lincoln, tgay1@unl.edu

Follow this and additional works at: https://digitalcommons.unl.edu/physicsgay

Part of the Physics Commons

Gay, Timothy J. , "Current Status Of The Saddle-Point Model" (1996). Timothy J. Gay Publications. 45. https://digitalcommons.unl.edu/physicsgay/45

This Article is brought to you for free and open access by the Research Papers in Physics and Astronomy at DigitalCommons@University of Nebraska - Lincoln. It has been accepted for inclusion in Timothy J. Gay Publications by an authorized administrator of DigitalCommons@University of Nebraska - Lincoln. 
Published in Two-Center Effects in Ion-Atom Collisions: A Symposium in Honor of M. Eugene Rudd; Lincoln, NE May 1994, edited by Timothy J. Gay and Anthony F. Starace. AIP Conference Proceedings 362; American Institute of Physics, Woodbury, New York, 1996. Pages 19-28.

Copyright (C) 1996 American Institute of Physics.

Used by permission. 


\title{
Current Status Of The Saddle-Point Model
}

\author{
T. J. Gay \\ Behlen Laboratory of Physics, University of Nebraska \\ Lincoln, Nebraska 68588-0111
}

\begin{abstract}
The current status of evidence for saddle-point electrons is discussed critically. Applications of the saddle-point model to the Barkas effect, ionizing collisions involving highly-charged projectiles, and proton-H collisions are considered.
\end{abstract}

\section{INTRODUCTION}

The saddle-point model of ionizing ion-atom collisions is based on the idea that when a charged projectile ionizes target electrons, some of the ejected electrons find themselves on the transient, moving saddle-point of Coulomb potential with a velocity that matches that of the saddle point. Feeling no force at this position, they "ride" the saddle out of the collision volume and are thus ionized. In the case of the prototypical $\mathrm{H}^{+}+\mathrm{H}$ system, or in any proton - neutral target collision, the saddle point moves at half the projectile velocity, so these electrons are often referred to as "v/2" electrons.

This model has been the topic of numerous papers [1-21], and has proven to be useful for the insights it provides into the general problem of collisional ionization. It has also been applied successfully in the analysis of scaling laws for prediction of total ionization cross sections $[6,19]$ and specific phenomena such as the Barkas effect [22-25]. But the model, its implications and interpretation, and some of the experimental evidence cited to support it have been controversial, and no consensus or comprehensive picture yet exists about the nature of saddle-point effects.

Prior to 1980, primarily as a result of the work of Rudd and co-workers [26], a standard view of ionizing collisions had developed. This view held that almost all the ionized electrons (excluding those produced in violent binary encounters) could be associated either with the ionized target or the receding projectile. The former, mostly having small momenta, were produced in "soft" collisions with the projectile and emerged almost isotropically from the collision region. (Electrons resulting from autoionization of the target were also included in this group.) The remaining ionized electrons had velocities very similar to that of the projectile, and formed a "cusp" distribution about its velocity vector in the forward direction. Based on these considerations, close-coupling calculations of ionization cross sections, for example, employed basis states that were centered either on the target, the projectile, or both. Born-approximation calculations, of course, are inherently one-center approaches to the ionization problem. 
In 1984, however, Winter and Lin [27] showed that their two-center closecoupling calculations of $\mathrm{H}^{+}+\mathrm{H}$ total ionization cross sections, which were much smaller than those measured experimentally below $25 \mathrm{keV}$, could be improved substantially by including basis states centered at a point mid-way between the two charge centers. This improvement implied that a large fraction of the ionized electrons were more appropriately associated not with the two charge centers but with the Coulomb saddle region of the collision. This result was consistent with an observation made earlier by Olson [28] that in classical trajectory Monte-Carlo (CTMC) calculations of ionizing collisions, an enhancement of electrons at v/2 was apparent. By 1985, various laboratories had begun to search for physical manifestations of "two-center effects," i.e. those in which ionized electrons felt roughly equal forces from each of the collision's charge centers. Conceptually, the simplest example of such an effect is the existence of saddle-point electrons.

\section{Evidence for Saddle-Point Electrons}

A brief critical analysis and overview of the various evidence for saddle-point electrons follows. The list is comprehensive, but not chronological. Not discussed is the work of Stolterfoht $e t$ al.[29], van der Straten and Morgenstern [30], Arcuni [31], and Swensen et al.[32]. These experiments dealt with departures from a Bornapproximation ionization picture[29] and post-collision interactions between electrons produced in target autoionization and the receding projectile[30-32]. They thus dealt with two-center effects, but not with saddle-point ionization.

\section{Forward-ejected electrons in slow $\mathrm{H}^{+}+H$ ionizing collisions}

Winter and Lin found that the disparity between their two-center expansion calculation and the measured cross sections increased with decreasing collision energy, implying that the "saddle-point cross section" will be largest for low energies. Indeed, Wannier threshold theory says that at the ionization threshold, all electrons will emerge on the saddle point [9]. The Winter and Lin work implies that the saddle-point mechanism will dominate other processes below $5 \mathrm{keV}$.

In an elegant experiment done at Utrecht and reported at this conference, Pieksma et al. $[14,20]$ have studied $\mathrm{H}^{+}+\mathrm{H}$ collisions between 1 and $6 \mathrm{keV}$, and found that $\mathrm{v} / 2$ electrons dominate the ejected electron spectra above $2 \mathrm{keV}$. An example of this is shown in Fig.1. While a classical interpretation of these results points directly to saddle-point emission, the quantum-mechanical view is more complicated, involving electron promotion through hidden crossings in the complex space of internuclear distance. The theoretical interpretation of the experimental results is complicated by contributions from "S," or "direct" ionization processes, and by the fact that the electron spectrometer used in these experiments detects electrons emitted into the full forward hemisphere. Nonetheless, the spectra display clear maxima at v/2 for incident proton energies above $2 \mathrm{keV}$ and would appear to be the cleanest signature of a saddle-point mechanism yet demonstrated. 


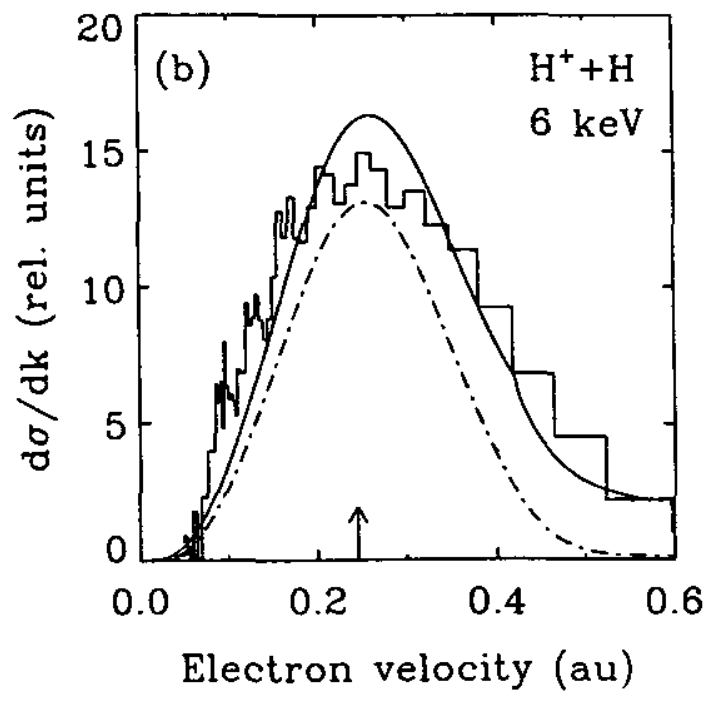

Figure 1. Relative electron yield vs. electron velocity for $6 \mathrm{keV} \mathrm{H}^{+}+\mathrm{H}$ collisions. Projectile velocity divided by two is indicated by the arrow. The dash-dot curve indicates the theoretical prediction for the saddle-point electron contribution; the solid curve is the total yield prediction, fitted with one height parameter to the experimental results. Data and theory of ref. [20].

\section{Saddle-point electrons at intermediate collision energies}

In 1986, Olson et al. [2] reported experimental data and CTMC calculations for doubly-differential electron ejection cross sections at $17^{\circ}$ in $\mathrm{H}^{+}+\mathrm{He}$ collisions. (It should be pointed out that these data were substantially similar to earlier measurements made by Rudd $e t$ al.) The incident energy range they studied was 60 to $200 \mathrm{keV}$. Plotting the data in velocity space, they showed that most of the electrons ejected in the forward direction have speeds roughly midway between zero and that of the projectile. Thus these electrons experience, over most of their ionizing trajectories, comparable forces from the $\mathrm{He}^{+}$ion and the receding proton. Olson $e t$ al. $[2,4]$ chose to call these mid-speed electrons "saddle-point" electrons because of their proximity, both in space and velocity space, to the actual Coulomb saddle point. By dividing the post-collision volume into three regions (see Table I), they were able to show that almost $60 \%$ of the ionized electrons are emitted in the middle saddle-point region for $60 \mathrm{keV}$ proton energy. 
TABLE I. Populations estimated using CTMC calculations (ref. 4) of the three spatial regions associated with the ionized target, Coulomb saddle point, and the receding projectile. Binning assignments were made when the projectile was at 50 a.u. 2 , with the $\mathrm{He}^{+}$at the origin. The boundaries of the three regions are the planes at $z=50 \times 1 / 3$ a.u. and $50 \times 2 / 3$ a.u. Antiproton projectile calculation are in parentheses.

\begin{tabular}{clll}
\hline & \multicolumn{3}{c}{ Flux fraction \% } \\
\cline { 2 - 4 }$E(\mathrm{keV})$ & $\begin{array}{l}\text { Target } \\
\text { region }\end{array}$ & $\begin{array}{l}\text { Midpoint } \\
\text { region }\end{array}$ & $\begin{array}{l}\text { Projectile } \\
\text { region }\end{array}$ \\
\hline 60 & $19.1 \pm 0.9$ & $57.8 \pm 1.5$ & $23.1 \pm 1.0$ \\
100 & $24.5 \pm 0.8$ & $59.4 \pm 1.3$ & $16.1 \pm 0.7$ \\
200 & $46.8 \pm 1.4$ & $47.6 \pm 1.4$ & $5.6 \pm 0.5$ \\
$(250)$ & $(67.8 \pm 2.8)$ & $(31.8 \pm 1.9)$ & $(0.5 \pm 0.2)$ \\
250 & $50.7 \pm 2.1$ & $45.9 \pm 2.1$ & $3.4 \pm 0.5$ \\
\hline
\end{tabular}

The major implication of this work was that the single-center view of ionizing collisions is incomplete and, in the energy range below about $150 \mathrm{keV}$, qualitatively misleading. An ancillary lesson was that plotting doubly-differential electron spectra in velocity space elucidates several key aspects of the ionization physics. This paper proved to be controversial for several reasons (see, e.g., references 10 and 15). First, the data exhibited no narrow spectral feature at $v / 2$, and the spectral maxima shifted from $0.85 \mathrm{v}$ to $0.3 \mathrm{v}$ as the proton energy increased over the energy range investigated. (A similar energy-dependant peak shift occurs in the data of Pieksma et al.[20]). Thus the objection was made that no clean signature of a saddle-point mechanism was apparent. This objection is valid, but can be resolved by reemphasizing that the papers of Pieksma et al.[20] and Olson et al.[2] are really claiming different things. At low energy a specific saddle-point mechanism has been identified and the spectra bear out the predictions of a calculation based on this mechanism. At the higher energies investigated by Olson et al., the picture is less precise, but is still based on the idea that most of the forward-ejected electrons are influenced about equally by both the target ion and the receding projectile, i.e. that they live in a region close to the saddle, and that this is apparent from the data once it is plotted in velocity space. A subset of these electrons (whose fraction grows smaller with increasing projectile energy) are those actually stranded on the saddle point and which have a speed precisely half that of the projectile.

A second objection was that by plotting the data in velocity space, mid-velocity maxima were artificially being introduced in the spectra $[10,12,13,15]$. For example, if one plots the differential cross sections for ejection at $90^{\circ}$ in velocity space, a maximum occurs (typically below $0.4 \mathrm{v} ;[15]$ ), but this maximum can hardly be attributed to saddle-point ionization. This concern has validity to the extent that one insists on identifying spectral maxima with a specific saddle-point mechanism. But such an identification is dubious at best in the intermediate energy regime. Nonetheless, if we ask the question "Where are most of the forward-ejected electrons (e.g., with $\theta \leq 15^{\circ}$ ) at, say $100 \mathrm{keV?",} \mathrm{the} \mathrm{answer} \mathrm{is:} \mathrm{roughly} \mathrm{midway}$ between the target and the projectile. This important point is obscured when the data are plotted differentially in energy or in velocity-vector space[12].

While ejected-electron spectra exhibit maxima at non-zero velocity values in 
velocity space at all ejection angles [18], maxima are only observed in the equivalent energy-differential spectra only for emission angles $\leq 15^{\circ}$ [4]. Gay et al. $[4,12]$ used this fact to answer objections of the second kind discussed above, claiming that since no kinematic effect was present in the energy-differential spectra, the existence of such maxima at the forward angles represented a "clear signature of saddle-point phenomena." Meckback et al. [15], however, have disagreed with this characterization, claiming instead that the energy maxima between $10^{\circ}$ and $15^{\circ}$ are simply the "backwash" or "remnants" of the charge-transfer-to-the-continuum (CTC) cusp. Herein lies a semantic difficulty. Any backwash from CTC would generally satisfy the rather general definition of saddle-point electrons as advanced by Olson $e t$ al. [2], but would not be characteristic of "true" $v / 2$ electrons that originate on or near the saddle-point.

But another question arises in this context. Visible on some $0^{\circ}$ spectra, both calculated and experimental, are secondary mid-velocity maxima below the CTC cusp. Such maxima cannot be the backwash of CTC, but could in turn be the saddle-point parents of the maxima at $10^{\circ}$. Unambiguous mid-velocity maxima in $\mathrm{d} \sigma /(\mathrm{dEd} \Omega)$ at $0^{\circ}$ would constitute strong evidence for the importance of saddle-point ionization, but not all spectra exhibit this effect. It is most obvious in the data of Gibson and Reid [33; see Fig.2 below], and in some CTMC calculations [figs. 2 and 3 of ref. 7]. It is also evident in some spectra from Meckback's group [see, e.g., Fig.5 of ref. 8; Fig.6 of ref. 18], but is absent from others. Experiments done at Nebraska by Gealy have failed to find it [34]. Experiments done at $0^{\circ}$ are difficult and beam contamination is a pernicious problem. Thus careful experiments need to be done to determine if this second maximum is real.

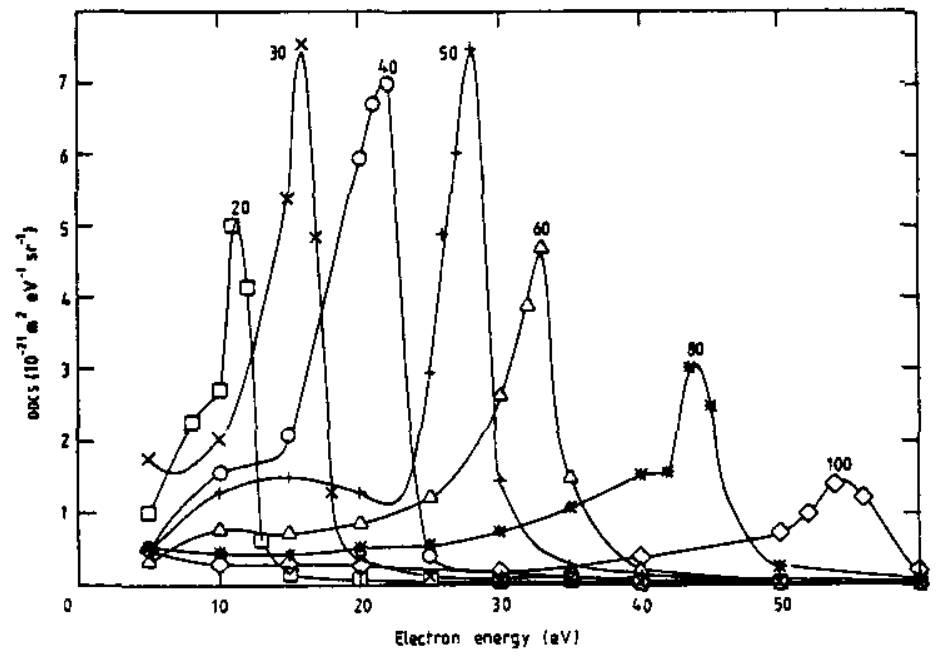

Figure 2. Doubly-differential cross sections in energy for $\phi^{\circ}$ emission of electrons in $\mathrm{H}^{*}+\mathrm{He}$ collisions at proton energies between $20 \mathrm{keV}$ and $100 \mathrm{keV}$ [33]. An apparent midvelocity secondary maximum, evident between 40 and $60 \mathrm{keV}$, disappears at higher energies, and, if present, is not resolved at lower ones. See also refs. 7,8, and 18. 


\section{Projectile charge-dependence of mid-velocity maxima.}

For a given projectile velocity $v$, the velocity of the saddle point depends on the final projectile $\left(\mathrm{Z}_{\mathrm{p}}\right)$ and target $\left(\mathrm{Z}_{\mathrm{l}}\right)$ charges as

$$
v_{S P}=\frac{v}{1+\left(Z_{p} / Z_{i}\right)^{1 / 2}}
$$

If saddle-point ionization is an important mechanism, one would expect the electron distribution maximum to shift for isotachic projectiles of different charge. Irby et al. [3] tested this idea using ${ }^{3} \mathrm{He}^{++}$and $\mathrm{H}^{+}$projectiles at specific energies from 60 to $120 \mathrm{keV} / \mathrm{amu}$. They obtained the somewhat counterintuitive result (albeit one predicted by eq.1) that the $\mathrm{He}^{++}$peak cross sections at $17^{\circ}$ occurred at lower velocities than did those for isotachic $\mathrm{H}^{+}$. The experimental situation is unclear on this point, however. Both the Argentinean group [10,13] and DuBois [17] have carefully remeasured these spectra and find no shift. On the other hand, Gay et al. [12] have also redone the experiment and confirm qualitatively that a shift is present. Moreover, Irby et al.[16], using $\mathrm{C}^{1+}, \mathrm{C}^{2+}$, and $\mathrm{C}^{3+}$ on $\mathrm{He}$ have seen chargedependent shifts, in the direction predicted by a saddle-point hypothesis, that have been qualitatively confirmed by DuBois[21].

Several points must be made here. First, neither CTMC [3] nor CDW-EIS [10] calculations predict a projectile-charge-dependent shift. If such an effect was truly present, one would certainly expect at least a fully classical calculation such as the CTMC to see it. It should also be noted, though, that Burgdörfer et al. $[5,11]$ have seen such shifts in their short-range model calculations in both one and three dimensions. Experimentally, Gay et al. [12] have shown how beam contamination could, in principle, produce an artificial shift, but measured the effect to be negligible for their data. DuBois [17] tried to mimic an artificial shift by intentionally contaminating his beam, but could not reproduce the results of Irby et al.[3] or Gay et al. [12]. Irby has pointed out that spurious scattering of electrons from analyzer back plates can mask shifts that are present [ref. 16 and these proceedings], but Bernardi and Meckback have shown that this effect is negligible in their case [35]. So the situation is confused.

The carbon-projectile experiments $[16,21]$ raise an interesting question about collisions in which at least one of the post-collision charge centers has at least one bound electron. If one makes the (only partially justified) assumption that no electrons are lost from the carbon, the shifts with projectile charge can be viewed in one of two ways. A saddle-point model simply explains them in terms of eq.1. But one can also argue that the changes in spectra are not peak shifts per se, but are caused by a reduction in the low-energy electron production cross sections as projectile charge is increased [see Fig.3 of ref.21]. This effect is caused by increased screening of the bare carbon nucleus as the net charge is decreased; with lower projectile charge the more sharply impulsive ionizing events will become more important relative to the "soft" large-impact-parameter collisions that dominate for higher charge states. 


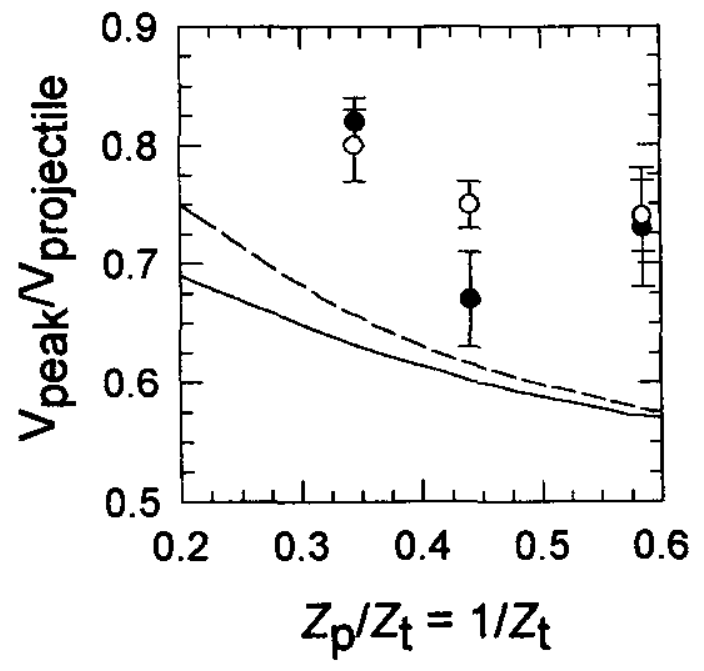

Figure 3. Position of peak in velocity space for $10^{\circ}$ emission in $50 \mathrm{keV}$ (open circles) and $100 \mathrm{keV}$ (solid circles) proton impact ionization. Left two data points are for Ne targets, the middle two are for $\mathrm{Ar}$, and the righthand two are for $\mathrm{He}, \mathrm{Z}_{\mathrm{p}}=1 ; \mathrm{Z}_{\mathrm{i}}$ is given by Slater's rules [36]. Solid curve corresponds to the geometric saddle-point velocity; the dashed curve is taken from the one-dimensional short-range potential calculation of ref. [5]. Data of ref. 12 (see text).

Let us consider, however, the experiments of Gay et al. [12] in which protons ionized $\mathrm{He}$, $\mathrm{Ne}$, and $\mathrm{Ar}$. Here the projectile charge remains constant, but an effective charge for the target becomes +1 only asymptotically. During the first stages of ionization, the different targets have different effective charges, which can be very cnudely estimated using Slater's rules [36]. (While effective charge of this type is really dependent on the distance between the escaping electron and the target, and on the momentum transfer to the ionized electron, any attempt to refine the effective charge concept is probably not warranted, given the crude nature of the model.) Thus Fig. 3 shows that target-dependent variations of the spectral maxima can be viewed as a saddle-point shift. The key point here, however, is that the screening model, used by DuBois [21] to explain the shifts in the carbon data, predicts shifts opposite those observed when applied to the (transient) effective charge of the target In summary, the saddle-point shift data at this point are inconclusive. An ideal experiment to test these ideas would involve ionization of atomic hydrogen by a series of bare projectiles at low impact velocities.

\section{Ridge electrons.}

In addition to the "longitudinal" evidence for saddle-point ionization provided by electrons emitted at small forward angles, there can also be "transverse" effects. 
Prior to the work of Olson et al.[2], Meckback et al.[1] presented the first detailed discussion of two-center effects with regard to mid-velocity electrons. Considering velocities in the region around $v / 2$ for forward-scattered electrons, they found cusps in the electron distribution at $0^{\circ}$ as the electron ejection angle was varied. They termed these electrons "Wannier-ridge" electrons, and attributed the ridge- or cusplike distribution to the transverse compression effect of the saddle region. The existence of a cusp, as such, at $0^{\circ}$ (as opposed to a smooth maximum) has since been called into question [37], and there is still controversy regarding this point. Moreover, the data presented by Meckback et al. was for $170 \mathrm{keV} \mathrm{H}^{+}+\mathrm{He}$ collisions, where it is unlikely that significant saddle-point ionization occurs. Nonetheless, analogous ridge-like features at $0^{\circ}$ (see, e.g., Fig. 8 of ref.[18]) are a general feature of ionized electron spectra, and can be attributed reasonably to transverse saddle-point focusing effects.

\section{Applications of the Saddle-Point Model}

We now consider three applications of the saddle-point model. The first has already been mentioned: the use of a third, mid-region expansion center in close coupling calculations of total ionization cross sections [27]. The fact that this technique is efficacious primarily at low energies, in conjunction with the Wannier threshold picture of saddle-point ionization, implies that "true" (mid-point stranding) saddle-point ionization can be a dominant mechanism only below $25 \mathrm{keV}$, at least for singly-charged projectiles [14]. The more general notion of mid-velocity ionization [2], however, can be used to understand total ionization cross section scaling laws and the Barkas effect.

\section{Ionization cross section scaling laws}

When highly-charged ions such as $\mathrm{C}^{9+}$ and $\mathrm{O}^{9+}$ ionize $\mathrm{H}$ or $\mathrm{He}$, it has been shown experimentally that the maximum values of the total ionization cross sections, $\sigma_{\max }$, and the incident projectile energies at which these cross sections occur, $E_{\max }$, are given by the scaling laws:

$$
\begin{aligned}
E_{\max } & =\mathrm{aq}{ }^{0.65} \times 10^{4} \mathrm{eV} / \mathrm{amu} \\
\text { and } \quad \sigma_{\max } & =\mathrm{bq}^{1.3} \times 10^{-16} \mathrm{~cm}^{2},
\end{aligned}
$$

where $\mathrm{q}$ is the projectile charge and $\mathrm{a}$ and $\mathrm{b}$ are phenomenologically determined constants [38]. These ad hoc equations have been used extensively in models of fusion plasmas. Using a simple, classical saddle point picture, Irby[6] was able to derive them from first principles. Essentially, he argued that at the energy where $\sigma_{\max }$ occurs, the saddle-point velocity ought to match the average target electron velocity. Moreover, the corresponding value of $\sigma_{\max }$ should be proportional to $\pi \mathrm{R}^{2}$, where $R$ is the distance between the saddle point and the target nucleus. This distance is determined in turn by picking the collisional impact parameter such that target electrons have enough energy to traverse the saddle region of the Coulomb potential. More recently, Janev et al.[19] have used the ideas of hidden crossings 
and superpromotion in saddle-point ionization to predict the specific energydependence of the ionization cross sections below their peak values.

\section{The Barkas effect}

Originally, the term "Barkas effect" was used to describe the fact that when $\mathrm{K}$ mesons decay in matter, the equally energetic $\pi^{+}$and $\pi^{*}$ particles produced in the decay travelled different distances before stopping [39]. More recently, the term has come to refer to the differences in electromagnetic interaction between a particle of matter and its environment as compared with its antimatter equivalent [25]. It is generally true that negatively-charged particles have lower stopping powers in matter than their positively-charged equivalents. At high energy, this has been attributed to a "polarization" effect, in which swift positive projectiles draw target electrons toward them, making ionization events more likely [39]. In this manner, the positively-charged particle loses energy more quickly and thus experiences a higher stopping power. This effect is typically less than $1 \%$. At lower energies, though, the ionization cross sections for, e.g., protons and antiprotons can be as large as $40 \%[22,23,40]$. Olson and co-workers [23-25] have shown that the difference in ionization cross sections for proton and antiproton projectiles is due almost entirely to production of electrons on the middle saddle region (see Table 1). Thus for initial energies of the order of $1 \mathrm{MeV}$ [41], the difference in proton and antiproton ranges is due primarily to saddlepoint effects. It is not clear for higher energies whether polarization or saddle-point effects dominate matter-antimatter range differences.

\section{ACKNOWLEDGEMENTS}

The author would like to thank H. G. Berry, J. Burgdörfer, R. D. DuBois, C. R. Garibotti, M. W. Gealy, V. D. Irby, R. E. Olson, C.O. Reinhold, and M. E. Rudd for numerous useful discussions of this topic. This work was supported by funding from the University of Missouri, the University of Nebraska, and the Department of Energy, Office of Fusion Energy (Grant \# DE-FG02-84ER53188).

\section{REFERENCES}

1. W. Meckbach, P. R. Focke, A. R. Goni, S. Suárez, J. Macek, and M. G. Menendez, Phys. Rev. Lett. 57, 1587 (1986).

2. R. E. Olson, T. J. Gay, H. G. Berry, E. B. Hale, and V. D. Irby, Phys. Rev. Lett. 59, 36 (1987).

3. V. D. Irby, T. J. Gay, J. Wm. Edwards, E. B. Hale, M. L. McKenzie, and R. E. Olson, Phys. Rev. A(RC) 37, 3612 (1988).

4. T. J. Gay, H. G. Berry, E. B. Hale, V. D. Irby, and R. E. Olson, Nucl. Instrum. and Meth. B31, 336 (1988).

5. J. Burgdörfer, J. Wang, and A. Bárány, Phys. Rev. A(RC) 38, 4919 (1988).

6. V. D. Irby, Phys. Rev. A 39, 54 (1989).

7. C. O. Reinhold and R. E. Olson, Phys. Rev. A 39, 3861 (1989). 
8. G. C. Bernardi, S. Suárez, P. D. Fainstein, C. R. Garibotti, W. Meckbach, and P. Focke, Phys. Rev. A 40, 6863 (1989).

9. G. Bandarage and R. Parson, Phys. Rev. A 41, 5878 (1990).

10. G. Bernardi, P. Fainstein, C. R. Garibotti, and S. Suárez, J. Phys. B 23, L139 (1990).

11. J. Wang, J. Burgdörfer, and A. Bárány, Phys. Rev. A 43, 4036, (1991).

12. T. J. Gay, M. W. Gealy, and M. E. Rudd, J. Phys. B 23, L823 (1990).

13. G. Bernardi, S. Suárez, P. Fainstein, C. Garibotti, W. Meckbach, and P. Focke, J. Phys. B 23, L829 (1990).

14. M. Pieksma and S. Y. Ovchinnikov, J. Phys. B 24, 2699 (1991).

15. W. Meckbach, S. Suárez, P. Focke, and G. Bernardi, J. Phys, B 24, 3763 (1991).

16. V. D. Irby, S. Datz, P. F. Dittner, N. L. Jones, H. F. Krause, and C. R. Vane, Phys. Rev. A 47, 2957 (1993).

17. R. D. DuBois, Phys. Rev. A 48, 1123 (1993).

18. S. Suárez, C. Garibotti, G. Bernardi, P. Focke, and W. Meckbach, Phys. Rev. A 48, 4339 (1993).

19. R. K. Janev, G. Ivanovski, and E. A. Solov'ev, Phys. Rev. A(RC) 49, R645 (1994).

20. M. Pieksma, S. Y. Ovchinnikov, J. van Eck, W. B. Westerveld, and A. Niehaus, Phys. Rev. Lett. 73, 46 (1994).

21. R. D. DuBois, Phys. Rev. A 50, 364 (1994).

22. R. E. Olson, Phys. Rev. A(RC) 36, 1519 (1987).

23. R. E. Olson and T. J. Gay, Phys. Rev. Lett. 61, 302 (1988).

24. T. J. Gay and R. E. Olson, Nucl. Instrum. and Meth. B40/41, 104 (1989).

25. D. R. Schultz, R. E. Olson, and C. O. Reinhold, J. Phys. B 24, 521 (1991).

26. See, e.g., M. E. Rudd, Y.-K. Kim, D. H. Madison, and T. J. Gay, Rev. Mod. Phys. 64, 441 (1992).

27. T. G. Winter and C. D. Lin, Phys. Rev. A 29, 3071 (1984).

28. R. E. Olson, Phys. Rev. A 27, 1871 (1983).

29. N. Stolterfoht, D. Schneider, J. Tanis, H. Altevogh, A. Salin, P. D. Fainstein, R. Rivarola, J. P. Grandin, J. N. Scheurer, S. Andriamonje, D. Bertault, and J. F. Chemin, Europhys. Lett. 4, 899 (1987).

30. P. van der Straten and R. Morgenstern, J. Phys. B 19, 1361 (1986).

31. P. Arcuni, Phys. Rev. A 33, 105 (1986).

32. K. Swenson, C. C. Havener, N. Stolterfoht, K. Sommer, and F. W. Meyer, Phys. Rev. Lett. 63, 35 (1989).

33. D. K. Gibson and I. D. Reid, J. Phys. B 19, 3265 (1986).

34. M. W. Gealy, private communication (1993).

35. G. Bernardi and W. Meckbach, Phys. Rev. A 51, 1709 (1995).

36. H. Eyring, J. Walter, and G. E. Kimball, Quantum Chemistry, (Wiley, New York, 1964) pps. 162-163.

37. G. Bemardi, S Suárez, P. Focke, and W. Meckbach, Nucl. Instrum. and Meth. B33, 321 (1988).

38. R. A. Phaneuf, R. K. Janev, and M. S. Pindzola, Oak Ridge National Laboratory Report ORNL-6090/V5 (Controlled Fusion Atomic Data Center, ORNL, 1987).

39. G. Basbas, Nucl. Instrum. and Meth. B4, 227 (1984).

40. P. D. Fainstein, V. H. Ponce, and R. D. Rivarola, Phys. Rev. A 36, 3639 (1987).

41. A. Adamo et al. (OBELIX Collaboration), Nucl. Phys. A558, 665c (1993). 